\title{
La cortesía valorizadora en las redes sociales. Análisis de un corpus de publicaciones en Facebook
}

\author{
JULia Vivas MárQuez \\ Universidad de Murcia \\ Facultad de Letras \\ C/ Santo Cristo 1 \\ 30001 Murcia \\ E-mail: julia.vivas@um.es
}

\section{FACE-FLATtering POLITENEsS in SOCIAL NeTWORKS. CORPUS ANALYSIS OF FACEBOOK POSTS}

ABSTRACT: This paper aims to conduct a pragmalinguistic analysis of face-flattering politeness strategies used by Facebook network users. For this purpose, a corpus of posts from twenty users from this social network has been analysed. The theoretical approach is based on face-flattering politeness as opposed to mitigating politeness. From this perspective, it has been intended to offer an analysis that takes into consideration both contextual conditions and features of this social network. It can also explain user's communicative behavior related to politeness strategies. As general conclusions it can be highlighted the prevalence of ritualized speech acts that reveal direct politeness, and also the indirect use of faceflattering politeness by showing interest, agreement and using jokes. tras de cortesia directa y el uso in directo de estrategias de cortesía valorizadora a través de manifestaciones de interés, acuerdo y bromas afiliadoras.

PALABRAS ClAVES: pragmática; ciberpragmática; cortesía; cortesía valorizadora; redes sociales.

SUMARIO: 1. Introducción. 2. La cortesía valorizadora. 3 . Análisis y resultados. 4. Conclusiones y futuras líneas de investigación.
SUMMARY: 1 . Introduction. 2 . Face-flattering politeness. 3. Analysis and Results. 4. Conclusions and future lines of research.

\section{LA POLITESSE VALORISANTE DANS LES RESEAUX SOCIAUX. ANALYSE D'UN CORPUS DE PU- BLICATIONS SUR FACEBOOK}

RÉSUMÉ: L'objectif de cet article est celui de réaliser une analyse pragmatique sur les stratégies et les ressources de la politesse valorisante employés par les utilisateurs du réseau social Facebook. Pour cela, nous avons pris comme corpus d'analyse les publications de vingt utilisateurs recueillies sur ce réseau social. Le cadre théorique de ce travail est basé sur la distinction entre la dite politesse valorisante et la politesse réparatrice; à partir de cette perspective, nous avons voulu offrir une analyse qui réponde aux caractéristiques et aux facteurs contextuels de ce réseau social et qui explique le comportement communicatif des utilisateurs en rapport avec les phénomènes de politesse. Comme principales conclusions nous pouvons signaler la prépondérance des actes de parole ritualisés comme preuves de politesse directe et l'usage indirect des stratégies de politesse valorisante à travers des collaborations thématiques.

MOTS CLÉS: pragmatique; cyberpragmatique; politesse; politesse valorisante; réseaux sociaux.

SOMMAIRE: 1 . 1. Introduction. 2 La politesse valorisante. 3. Analyse et résultats. 4. Conclusions et futures lignes d'investigation.

$\begin{array}{ll}\text { Fecha de Recepción } & 22 / 01 / 2014 \\ \text { Fecha de Revisión } & 21 / 08 / 2014 \\ \text { Fecha de Aceptación } & 10 / 09 / 2014 \\ \text { Fecha de Publicación } & 01 / 12 / 2014\end{array}$




\title{
La cortesia valorizadora en las redes sociales. Análisis de un corpus de publicaciones en Facebook
}

\author{
NOMBRE Y APELLIDOS
}

\section{INTRODUCCIÓN}

La ingente expansión de redes sociales en los últimos años ha constituido uno de los fenómenos más significativos en el cambio de modelo de comunicación que se ha producido en internet. La presencia de estas plataformas de interacción juega un papel fundamental en lo que autores como Yus denominan "un marco creciente de hibridación o amalgama de redes sociales físico-virtuales" (2010: 141), en el que los usuarios manejan sus relaciones interpersonales.

El caso de la red social Facebook -de donde se ha extraído el corpus de este trabajo- supone una plataforma de interacción en internet en la que prima claramente una función social por encima de una función informativa. A diferencia de otros medios, como los blogs, o los foros de opinión, el objetivo primordial de los participantes en esta red es la mera comunicación con la finalidad de establecer o mantener relaciones sociales.

En este sentido, y en relación al uso de estas redes, apunta Miller (2008: 393-395, ápud Yus, 2010: 151) que se ha producido:

[...] un cambio de énfasis desde la tecnología del blog, que propugnaba la creación de contenidos más allá de la interacción, a los perfiles de las redes sociales que enfatizan la interacción en red sobre el texto interesante [...] la sociabilidad sin el intercambio de información real [...] hacia lo que se está denominando "tecnologias fáticas", tecnologías que construyen relaciones y sostienen la interacción social mediante el contacto y la intimidad continuos (pero no informativos).

Los usuarios de estas redes, por tanto, encuentran en este contexto un marco abierto para el mantenimiento y el refuerzo de las relaciones interpersonales, para lo cual hacen un uso -a menudo exagerado- de estrategias de cortesía valorizadora. A esto hay que añadir el hecho de que, como subraya Yus:

En Internet existe, además, una ausencia de pistas contextuales que normalmente facilitan, en entornos conversacionales fisicos, la elección de una determinada estrategia. Este hecho puede desembocar en una sobreabundancia de expresiones manifiestas de cortesia (2010: 295).

Por otro lado, aunque las razones que determinan el uso de esta red social pueden ser variadas (acceder a otras fuentes de información, por ejemplo), 
los usuarios, normalmente, esperan algún tipo de recompensa por su utilización, y asumen que esta recompensa suele revertir en el refuerzo de su propia imagen, de ahí que, una vez más, este medio de comunicación presente un uso que va siendo cada vez más convencionalizado de estrategias de cortesía valorizadora.

Hay que tener en cuenta, sin embargo, que el caso de Facebook constituye una plataforma donde se combinan distintos tipos de canales de interacción, como puede ser correo electrónico (mensajes personales), foros de opinión (grupos), chats, micro-blogs (notas y publicaciones en el muro), y por supuesto, no todas obedecen a los mismos propósitos. Este trabajo se centra en analizar las estrategias de cortesía más frecuentes en las publicaciones del muro de los participantes, que tienen una visualización semipública (dependiendo de las opciones de privacidad elegidas por el usuario).

El corpus objeto de estudio está compuesto por publicaciones que aparecen en la red social Facebook entre los años 2009 y 2013. Dichas muestras -como se ha señalado- se ubican en el muro de los usuarios seleccionados con una visibilidad abierta a los amigos de los usuarios elegidos; es decir, no estaban restringidas a determinadas personas. Los perfiles corresponden a veinte personas - diez hombres y diez mujeres- con edades comprendidas entre los 25 y los 35 años, de nacionalidad española; todos ellos han concluido estudios universitarios.

Entre las cuestiones más significativas que se han de tener en cuenta a la hora de analizar la cortesía en internet y, en concreto en la red social Facebook, hay que considerar:

1. La ausencia de índices contextuales, que repercute, entre otros aspectos, en la inexistencia de comportamiento no verbal ni paraverbal. Aunque esta se puede suplir con determinados recursos tipográficos indicadores de la actitud del hablante (mayúsculas, onomatopeyas, juegos tipográficos, signos de entonación, emoticonos, etc.), hay que recordar que este medio carece de una comunicación no verbal no intencionada.

2. La asincronía, que influye directamente en el intercambio de turnos y en el número de interlocutores; ambos se pueden prolongar sin limitaciones espacio-temporales.

3. El uso del lenguaje responde, en la mayoría de los casos, a lo que se denomina "discurso escrito oralizado", cuyas características reflejan un empleo consciente de marcas de oralidad.

Por último, uno de los factores que más parece influir a la hora de considerar la cortesía en esta red social es el propio concepto de "amigo" que aúna distintos tipos de relación interpersonal. Para Cambra González: 
[...] este efecto de sesgo que se opera al asimilar a toda persona vinculada en $\mathrm{Fa}$ cebook a una misma categoria operativa no es sino un primer paso que motiva la irrupción de ciertos efectos distorsionadores que la socialización a través de este medio introduce en las relaciones personales (2009: 155).

\section{LA CORTESÍA VALORIZADORA}

En líneas generales, las revisiones teóricas sobre los estudios de cortesía coinciden en establecer su punto de partida en los trabajos de Goffman (1971 [1959], 1970 [1967]), a los que suceden las aportaciones de Lakoff (1973), Leech (1983) y Brown y Levinson $(1978,1987)$. A pesar de las numerosas discrepancias y las alternativas que se han ofrecido al modelo teórico de estos dos últimos autores, su teoría sobre la imagen y la cortesía se sigue considerando la más elaborada hasta el momento.

Una de las críticas más difundidas sobre el modelo de cortesía propuesto por Brown y Levinson $(1978,1987)$, además de su etnocentrismo cultural, ha sido la de dar una visión demasiado pesimista de la interacción humana, y presentar esta como una constante lucha de amenazas. Así, autores como Locher (2004: 74-75, ápud Barros García, 2011: 125) consideran como inconveniente de esta teoria el hecho de que se desarrolle teniendo en cuenta solo una de las funciones de la cortesía, la mitigadora o reparadora, obviando el carácter social o relacional de la misma.

Leech (1983: 104-105) realiza una temprana alusión a esta cuestión al referirse a determinados tipos de actos corteses en los que no hay lugar a amenazas. En su trabajo, el autor distingue entre cuatro clases de funciones ilocutivas según su relación con los objetivos sociales (competitiva, sociable, colaboradora y conflictiva). En esta clasificación, la función sociable (convivial en inglés) se refiere a actos considerados intrínsecamente corteses como pueden ser felicitar o agradecer.

Son varios los investigadores que coinciden en subrayar esta misma idea, como Lavandera (1988), Bayraktaroglu (1991) o Sifianou (1995), entre otros ${ }^{1}$. Entre las propuestas que más aceptación han tenido en relación a este tema se encuentra la de Kerbrat-Orecchioni. Para esta autora (2004: 43), la cortesía, además de mitigar amenazas, consistiría también y, sobre todo, en lo contrario, en producir "anti-amenzas"; es decir, actos de habla valorizadores o halagadores de la imagen positiva del destinatario, como pueden ser el agradecimiento o el cumplido.

\footnotetext{
1 Además, autores como Meier (1995) consideran incluso dudosa la propia definición que constituye un FTA. Por ejemplo, en las peticiones y los consejos, puesto que podrian imaginarse muchos contextos en los que estos mismos actos actúan como una señal de solidaridad: el hablante puede utilizarlos para ayudar a su interlocutor y no para entrometerse en su espacio personal o en su libertad de acción (Meier, 1995, ápud Barros Garcia, 2011: 110).
} 
En consecuencia, Kerbrat-Orecchioni (1992, 1997, 2004) defiende que es fundamental un marco teórico para estos actos que considera como la pendiente positiva de los FTAs (face-threatening acts) y propone denominarlos FFAs (face flattering acts), actos "agradadores" o "halagadores" de la imagen $^{2}$. Partiendo de esta propuesta, todo acto de habla puede ser descrito como un FTA o un FFA, o un complejo de estos dos. De esta manera, pueden distinguirse dos formas de cortesía:

1. Cortesía negativa: consiste en evitar un FTA o suavizar su realización; adquiere un perfil abstencionista.

2. Cortesia positiva: consiste en realizar un FFA de preferencia reforzado; adquiere un perfil produccionista.

De este modo, para la citada investigadora en las interacciones se produciría siempre un juego de balanzas entre FTA y FFA, y la tendencia general, en su opinión, es que en situaciones no conflictivas "los FTAs tienden ampliamente a ser suavizados, mientras que los FFAs, por el contrario tienden a ser reforzados" (2004: 45).

Este mismo concepto, o similar, ha sido tratado por otros autores bajo diferentes denominaciones. Así, por ejemplo, Bayraktaroglu (1991) se refiere a este tipo de actos como Face Boosting Acts (FBAs) "actos levantadores / aumentadores de la imagen", que tienen como objetivo satisfacer las necesidades de imagen del hablante y/o destinatario. Sifianou (1995) opta por el término Face Enhancing Acts (FEAs) “actos realzadores de la imagen” incluyendo en estos actos ofrecer, felicitar, halagar, etc.; a esta misma denominación también se acogen autores como Terkourafi (2005) y Leech (2007) ${ }^{3}$.

Esta perspectiva ha sido la adoptada por numerosos estudios dedicados al análisis de la cortesía en español (Carrasco Santana, 1999, Hernández Flores, 2002, 2004, Albelda, 2003, 2004, 2005, Bernal, 2005, 2007 o Barros García, 2011, entre otros). Todos ellos coinciden, de alguna manera, en subrayar la idea de que la cultura española tiende a la valorización cortés, y de que esta función de la cortesía, con respecto a la mitigación de amenazas, no resulta secundaria.

En esta linea, por ejemplo, Carrasco Santana (1999: 15), en una revisión del modelo de Brown y Levinson, opta por utilizar los términos "cortesía valorizante" y "cortesía mitigadora" en lugar de cortesía positiva y negativa. La cortesía valorizante hace alusión a los actos de habla que buscan favorecer la imagen del interlocutor. En palabras del autor:

\footnotetext{
2 La traducción es de la autora.

${ }^{3}$ Las traducciones de los términos han sido tomadas del trabajo de Barros García (2011:127).
} 
La cortesía valorizante supone la realización de actos corteses de acuerdo con las normas de comportamiento mejor valoradas socialmente en la relación con los demás, para lograr que ésta sea armoniosa y que nuestra imagen personal se vea reconocida (1999: 22).

También Hernández Flores, en su trabajo sobre la cortesía como búsqueda del equilibrio de la imagen social, se refiere a ella como "realce de la imagen sin la presencia de amenazas" y la considera como una de las tres funciones atribuidas a la cortesía, junto con la "atenuación de posibles riesgos para la imagen" y la "reparación de posibles daños" (2004: 101).

Albelda $(2004,2005)$ se acoge al término "cortesía valorizante" y subraya el predominio de este tipo de estrategias en el español peninsular por encima de las de carácter mitigador. Bernal (2007) utiliza esta misma denominación y la define como una cortesía que tiene por objetivo "valorar positivamente al interlocutor con actividades como hacerle quedar bien, halagarle explícitamente con cumplidos a su persona" (2007: 127) y constata la alta frecuencia de este tipo de actividades en su corpus de análisis.

Por su parte, Barros García (2011) opta por el término "cortesía valorizadora" frente a "valorizante" ${ }^{4}$, definiéndola en estos términos:

[...] actividad cortés enfocada hacia la producción de algún tipo de beneficio para la imagen del interlocutor, beneficio que suele tener consecuencias positivas para la imagen del propio hablante y que contribuye a la creación de lazos interpersonales más sólidos entre los interlocutores y al aumento de la eficacia comunicativa (2011: 136).

Su análisis sobre las funciones y estrategias relacionadas con esta en la conversación coloquial confirma el predominio de la cortesía valorizadora en la sociedad española.

\section{ANÁLISIS Y RESULTADOS}

El análisis cualitativo que se ha llevado a cabo en este trabajo para indagar en los actos de habla valorizadores de Facebook está basado en la taxonomía propuesta por Albelda (2005) sobre los actos de refuerzo de la imagen, utilizada también por Barros García (2011); esta clasificación distingue entre valorización cortés directa y valorización cortés indirecta.

\subsection{VALORIZACIÓN CORTÉS DIRECTA}

4 Para una revisión bibliográfica sobre el estado de la cuestión sobre la cortesía en el mundo hispánico se puede consultar la tesis doctoral de Barros García (2011). 
Este tipo de cortesía coincide con la propuesta -explicada en el apartado precedente- de Kerbrat-Orecchioni sobre los "actos agradadores de la imagen" o Face Flattering Acts (FFAs). Este tipo de estrategias comunicativas se destinan, por tanto, a reforzar la imagen de los interlocutores.

Para la clasificación de este tipo de actos, en este análisis, se ha tomado como referencia la distinción formulada por Haverkate (1994) sobre los actos de habla corteses ${ }^{5}$ en relación con su frecuencia de aparición en las publicaciones del corpus; este autor establece la siguiente división:

1. Actos de habla expresivos, cuyo objetivo ilocutivo puede definirse como la expresión de un estado psicológico del hablante, causado por un cambio, que atañe al interlocutor o a él personalmente. Son actos centrados en el oyente (Haverkate, 1994: 80). Los más significativos en este corpus son: felicitaciones, agradecimientos, saludos, cumplidos, expresión de buenos deseos y expresiones de apoyo y de ánimo.

2. Actos de habla comisivos, cuyo objetivo ilocutivo se define como la expresión de la intención del hablante de realizar, en beneficio del oyente, la acción descrita por el contenido preposicional (Haverkate, 1994: 106). La invitación se perfila como un acto prototípico de esta categoría en el corpus analizado.

Debido al elevado número de publicaciones e intervenciones presentes en el corpus, se recoge una selección representativa de algunos de los enunciados considerados más significativos para cada categoria:

\begin{tabular}{|c|c|}
\hline \multicolumn{2}{|r|}{ ACTOS DE HABLA EXPRESIVOS } \\
\hline FELICITACIONES & 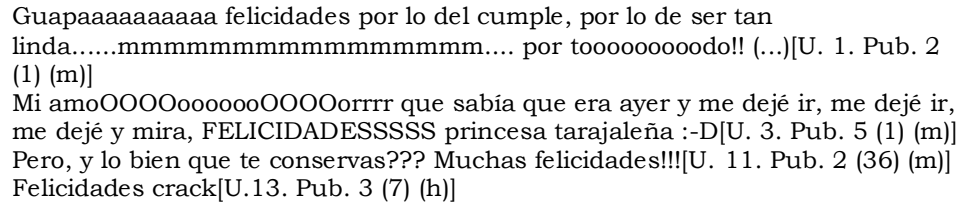 \\
\hline $\begin{array}{l}\text { CUMPLIDOS Y PIRO- } \\
\text { POS }\end{array}$ & $\begin{array}{l}\text { qué mona eres, y qué linda! y cómo te entiendo![U. } 7 . \text { Pub. } 2(2)(\mathrm{m})] \\
\text { Y tú, qué bonita eres tú.[U. } 8 \text {. Pub. } 5(2)(\mathrm{h})] \\
\text { Compadre....tu haces algo feo?......(a ver que me vas a contestar eh?)...(..)[U. } \\
11 . \text { Pub. } 10(3)(h)] \\
\text { guapo pijo!! :)[U. } 19 . \text { Pub. } 3(1)(\mathrm{m})] \\
\text { Dos besos te daba!!! Dos besos!![U. 19. Pub. } 5(2)(\mathrm{h})] \\
\text { Olé ahí[U. } 20 . \text { Pub. } 2(2)(\mathrm{m})]\end{array}$ \\
\hline SALUDOS & $\begin{array}{l}\text { Eeeeee! Ya estás por aki? Es posible que vaya la semana q viene a Badajoz. To- } \\
\text { davia no lo he decidido. Estarás? Besito[U. } 4 \text {. Pub. } 7(1)(\mathrm{m})] \\
\text { hla guapa!te exo de menos!!!estaras super bien no??t kero!![U. } 7 \text {. Pub. } 8(1)(\mathrm{m})] \\
\text { Hola tesoro, nos vemos enseguida! Muak [U. 10. Pub. } 2 \text { (4) (m)] } \\
\text { Eres mi amigo 60; yujuu! Qué tal va todo? Besazos[U. 15. Pub. } 3 \text { (1) (m)] }\end{array}$ \\
\hline
\end{tabular}

5 Haverkate (1994: 77) toma como referencia la tipología de los actos de habla de Searle (1976). 


\begin{tabular}{|c|c|}
\hline $\begin{array}{l}\text { EXPRESIÓN DE ÁNIMO } \\
\text { Y DE BUENOS DESEOS }\end{array}$ & $\begin{array}{l}\text { Que tengáis una buena celebración!!! Besosssss[U. 5. Pub. } 4(4)(\mathrm{m})] \\
\text { Eso es saber vivir, y no lo del doctor Torreiglesias. Disfruta las vacaciones!!![U. } \\
5 \text {. Pub. } 11(5)(\mathrm{h})] \\
\text { Ánimo!, si yo lo he dejado (que me fumaba un paquete y medio diario), tú tam- } \\
\text { bién puedes. [U. } 8 \text {. Pub. } 6(4)(\mathrm{m})] \\
\text { vamos tio. animo. fuerza. te mando mi apoyo. un abrazo campeón[U.14. Pub. } 2 \\
\begin{array}{ll}\text { (9) (h)] } \\
\text { Genial!!!Ahora a recuperarse pronto!!![U. 20. Pub. } 1 \text { (6) (m)] }\end{array}\end{array}$ \\
\hline AGRADECIMIENTOS & $\begin{array}{l}\text { Thank you so much, moito obrigada, graciñas y, en definitiva... muchísimas } \\
\text { gracias a tod@s... Será un dia genial! Y mañana, y pasado, y el fin de semana!!! } \\
\text { Mis cumples son laaaargos![U. 1. Pub. } 5(1)(\mathrm{m})] \\
\text { CHICOS MUCHÍSMIAS GRACIAS A TODOS, ASÍ ES MUCHO MÁS FÁCIL PA- } \\
\text { SAR A LOS 30!!! UN BESICO MU FUERTE A TOD@S! [U. 14. Pub. } 1 \text { (1) (h)] } \\
\text { No me lo puedo creer: he tenido tiempo de dibujar... ¡más de una hora sin pa- } \\
\text { rar! Doy gracias al cielo y a todos los que han creído en mi todos estos años.[U. } \\
\text { 18. Pub. } 4 \text { (1) (h)] } \\
\text { Muchas Gracias por el mensajito!!!! no sabes que alegria me dio verte por } \\
\text { alli!!!!!! Un abrazo enorme!!!!!U. 20. Pub. } 4(1)(\mathrm{m})]\end{array}$ \\
\hline
\end{tabular}

\begin{tabular}{|c|c|}
\hline \multicolumn{2}{|r|}{ ACTOS DE HABLA COMISIVOS } \\
\hline $\begin{array}{l}\text { INVITACIONES Y OFRE- } \\
\text { CIMIENTOS }\end{array}$ & $\begin{array}{l}\text { Ey!!! A ver si quedamos un dia para tomar algo, que hace mil que no sé nada } \\
\text { de tu vida!!! [U. 2. Pub. } 6(3)(\mathrm{h})] \\
\text { (...) Quieres unos bocatas el jueves en la codosera o x esa zona en la pis- } \\
\text { cina?? Reservanos un dia porfi!!![U.7. Pub. } 7 .(3)(\mathrm{m})] \\
\text { (...), ¿Qué tal estás? ¿Cuándo quedamos para una terracita, piscina, café, } \\
\text { cine...? Besitos. [U. 9. Pub. } 6(1)(\mathrm{m})] \\
\text { Hola!! Una cerve para cuando?[U. 15. Pub. } 2 \text { (1) (m)] }\end{array}$ \\
\hline
\end{tabular}

TABLA 1. EJEMPLOS DE VALORIZACIÓN CORTÉS DIRECTA

En líneas generales, estos de actos de habla presentan recursos de intensificación muy marcados que redundan en la valorización cortés del enunciado. Se señalan, a continuación, los procedimientos de intensificación más significativos:

1. Procedimientos lingüísticos:

1. 1. Recursos léxico-semánticos:

- Adjetivos y sustantivos valorizantes: crack; guapo; lindas; tesoro.

- Interjecciones de júbilo y sorpresa: Olé ahí!; hey wapis!

- Construcciones hiperbólicas: millones de gracias.

- Interrogaciones retóricas con fines intensificadores: $y$ lo bien que te conservas???; tu haces algo feo?

1. 2. Recursos morfológicos:

- Adjetivos en grado superlativo: Wapisssimmmmaaaa.

- Prefijos intensificadores: super bien.

- Sufijos diminutivos y aumentativos apreciativos: bonico; pequeñuela; prontito; besazo.

1. 3. Recursos sintácticos: 
- Topicalizaciones y dislocaciones: Dos besos te daba!!! Dos besos!!; Una cerve para cuando?; Eso es saber vivir.

- Conectores pragmáticos que intensifican los enunciados: ya verás como si.

- Enunciados exclamativos: ¡qué grande eres!; qué mona eres, y qué linda y cómo te entiendo!

- Uso de la deixis personal con fines intensificadores: tú puedes con eso; Y tú, qué bonita eres tú.

2. Procedimientos paralingüísticos:

- Repetición de vocales y consonantes por alargamiento: Guapaaaaaaaaaa; amoOOOOoooooOOOOorrr.

- Signos de exclamación e interrogación repetidos: enhorabuena!!!!!!

- Uso de mayúsculas: ME ENCANTAAAAAAAAAAAAAAAAAAAAAA

- Emoticonos: guapo pijo!! :)

A la intensificación habitual de este tipo de actos de habla, se añade el hecho de que se trata de un discurso mediado por ordenador u otros dispositivos electrónicos, lo que hace que estos enunciados se carguen de marcas de oralidad que a menudo pueden resultar redundantes.

Por otra parte, hay que considerar que las manifestaciones de cortesía positiva más frecuentes son aquellas que resultan de alguna manera "ritualizadas"; esto es, aquellas definidas como estrategias de cortesía directa (felicitaciones, cumplidos, muestras de ánimo, apoyo, etc.); todos estos actos de habla son -en lineas generales- inherentemente corteses y no suponen ningún riesgo de amenaza para la imagen del oyente. Ello explica que sea esta tipología de actos de habla los más frecuentes en la red social de Facebook, cuyo fin es el mantenimiento de las relaciones interpersonales.

Como señala Barros García, este tipo de valorización cortés "comporta un mayor grado de ritualización que la valorización cortés indirecta, especialmente en lo que se refiere a las conversaciones con respecto a la situación en la que tiene lugar la comunicación" (2012: 110). En el caso de Facebook, los participantes carecen de un contexto fisico que determine este comportamiento, pero parecen asociar esta red social con determinadas situaciones comunicativas frecuentemente ritualizadas (como pueden ser los cumpleaños, por ejemplo).

Uno de los factores que propician este tipo de actividad cortés es el carácter semi-público de las publicaciones en el muro, unido al hecho de que se trata de mensajes escritos que pueden permanecer por tiempo indefinido en la página del perfil de los usuarios. Su uso también guarda relación con 
el fenómeno particular de esta red social que es el concepto de "amigo" al que se ha hecho mención en la introducción. A menudo, este término aúna a conocidos de los usuarios sin que exista entre ellos una relación de confianza, lo cual incide en el número de actos de habla ritualizados, frecuentes también en determinados contextos físicos en la sociedad española donde los hablantes actúan para favorecer la cordialidad y donde prevalece el contenido ilocutivo por encima de la expresión lingüística en sí.

\subsection{VALORIZACIÓN CORTÉS INDIRECTA}

Aunque a primera vista estas actividades no parecen afectar directamente a la imagen del interlocutor -como sucede en las anteriores- sí es cierto que, de diferentes maneras, tienden a fortalecer la imagen de los participantes, así como sus relaciones interpersonales. Bernal, hablando de la cortesía temática, las define del siguiente modo:

[A]ctividades insertas en el discurso social plasmado en la conversación [que] encarnan una actividad de mostrar interés y afectan a la imagen de los participantes y a su relación interpersonal. De ahí que afirmemos que tienen un efecto social de cortesía (Bernal, 2007: 161) ${ }^{6}$.

Entre este tipo de actividades se encuentran las colaboraciones temáticas, que son las más frecuentes entre las intervenciones de los participantes de esta red social. La interpretación cortés de estas intervenciones dependerá, en la mayoría de los casos, del contexto y la situación en la que se hallen los participantes. Sin embargo, en relación al corpus analizado, hay que considerar que la influencia de la situación comunicativa es muy tenue, puesto que se trata de interacciones comunicativas mediadas por tecnologias digitales en las que los usuarios no se ven ante la situación de tener que colaborar en la conversación o de regular los intercambios conversacionales. Por tanto, en este análisis se consideran como una muestra de cortesía.

Las colaboraciones temáticas que más se repiten en el corpus suelen ser de tipo reactivo ante una publicación hecha por el usuario de un perfil en su propio muro. En menor medida, se encuentran ejemplos de carácter iniciativo que suelen relacionarse con muestras de interés hacia algún aspecto de la vida del usuario de un perfil de Facebook. Por limitaciones de espacio se recogen tres de las estrategias más frecuentes a la hora de mostrar cortesía valorizadora indirecta en el muro de esta red social: las manifestaciones de interés, las manifestaciones de acuerdo y las bromas afiliadoras.

6 En cursiva en el original. 


\subsubsection{MANIFESTACIONES DE INTERÉS}

La ausencia de índices contextuales hace que frecuentemente los usuarios de Facebook recurran a muestras de interés explícitas como reacción a publicaciones de otros usuarios.

En la siguiente publicación participan cuatro personas. Las intervenciones (2) y (3) constituyen ejemplos de interés explícito a través de signos de interrogación y una pregunta. La respuesta del usuario 19 incentiva una nueva muestra de interés esta vez por su afición a un programa de televisión. La última intervención, a modo de contestación, supone un intento de no dañar su imagen (por las posibles connotaciones que pudiera tener este programa entre sus amigos):

[1]

(1) Usuario 19: Muy mal, Bisbal.

(2) Amigo $1(\mathrm{~m})$ : ????

(3) Amigo $2(\mathrm{~m})$ : Que ha liado el bisbi??

(4) Usuario 19: Ha dejado escapar a una Jurado de su equipo para quedarse con una Pantoja.

(5) Amigo $3(\mathrm{~m})$ : Jajjaja pero bueno te picaste con la Voz?

(6) Usuario 19: Un poco sí, pero como sigan largando a los mejores me voy a desenganchar pronto... [U. 19 (h). Pub. 1]

A menudo, son los propios usuarios los que parecen buscar las manifestaciones de interés por parte de sus amigos, sabiendo que estas generan un refuerzo para su propia imagen; así, recurren a publicaciones de enunciados de interpretación abierta, como sucede en el siguiente ejemplo:

[2]

(1) Usuario 7: la vida te lleva por caminos raros...

(2) Amigo $1(\mathrm{~m}):$ ¿?

(3) Amigo 2 (h): Al final tas metio al mundo del porno aleman*?????ya era hora hija.....yevamos años esperandolo....jejejeje

(4) Amigo 2 (h): Ya fuera de coña, what happen mozita????

(5) Usuario 7: pues aún estoy en modo espera... a ver qué pasa!

(6) Usuario 7: (alude a amigo 2) menudo comentario!!!

(7) Amigo $3(\mathrm{~m})$ : Cuando nos vemos guapa!?! Por donde estas?! [U. 7 (m). Pub. 3]

La publicación del usuario 7 genera tres intervenciones en forma de preguntas interesándose por el significado del enunciado y por el estado del usuario. La intervención (3), a través del humor, refuerza la imagen de afiliación de los participantes. La respuesta del usuario 7 deja entender que se 
trata de un asunto de su vida del que están al tanto las personas que participan y determinados amigos, lo que refuerza una vez más la imagen de afiliación. La última intervención queda al margen del tema que inicia la publicación, pero supone una nueva muestra de cortesía a través de una invitación indirecta y una nueva manifestación de interés por la vida del usuario 7.

\subsubsection{MANIFESTACIONES DE ACUERDO}

Son, con gran diferencia, más frecuentes que las manifestaciones de desacuerdo en esta red social. Una de las razones parece obedecer a las características del contexto que ya se han comentado previamente. Cuando un usuario comenta algo en el muro de otro usuario es de alguna manera consciente de que está interviniendo en un espacio ajeno que no es completamente público. Por este motivo, los muros de esta red social contienen muchas menos expresiones de descortesía que otras plataformas como pueden ser Twitter, determinados foros de opinión o incluso perfiles públicos de Facebook.

En el siguiente ejemplo, el usuario 2 muestra su opinión ante la concesión del premio Nobel de la Paz a la UE; diferentes recursos tipográficos enfatizan su indignación ante este hecho:

[3]

(1) Usuario 2: ¿El premio Nobel de la QUÉEEEEE? ¿Cómooooo? Permiso para reirme...

(2) Amigo 1 (m): o para llorar...lo que te pida el cuerpo!

(3) Amigo $2(\mathrm{~m})$ : Permiso para meter la cabeza bajo tierra por vergüenza ajena...

(...)

(6) Amigo $6(\mathrm{~m})$ : Podian habernos dado el de economía....

(...)

(8) Amigo 7 (h, extranjero): permission granted

(9) Amigo 8 (h): ¿Y qué nos sorprende? ¿No le dieron a Obama el mismo galardón ocupando tropas gringas Afganistán (por decir lo menos)? •

(10) Amigo 9 (m): rie, rie, q es para mondarse •

(...) [U. 2 (m). Pub. 4]

Los comentarios que siguen a la publicación muestran su acuerdo con las palabras del usuario 2. Así, las intervenciones 2, 3, 8 y 10 colaboran en la petición irónica que supone el enunciado "permiso para reírme...". Con la intervención 9, el amigo 2 acude a la primera persona del plural para reforzar la muestra de acuerdo de todos los participantes. 
Por otra parte, es frecuente que los usuarios recurran a preguntas abiertas que buscan generar intervenciones de sus amigos:

[4]

(1) Usuario 12: Se está poniendo la cosa pa ir quemando cosicas no?

(2) Amigo 1 (m): Empezando por los politicos (...) [U.12 (h). Pub. 2]

No se trata, en estos casos, de peticiones de información, sino de preguntas retóricas que muestran una opinión. En el ejemplo siguiente, el usuario 17 expone su preocupación ante el aumento de horas lectivas por parte de la administración. Las intervenciones que le siguen confirman su acuerdo con este hecho colaborando a través de respuestas y argumentos a la pregunta formulada por el usuario 17.

[5]

(1) Usuario 17:

profesores de Castilla y León, bienvenidos oficialmente al aumento de horas lectivas... ¿qué será lo próximo?

(2) Amigo 1 (h): Sin disimulos, poner el culo...

(3) Amigo 2 (m): Lo próximo será que la gente cualificada se canse de que no les valoren y haya una fuga de cerebros a otros paises. Què verguenza!

(4) Amigo $3(\mathrm{~m})$ : ... se me ocurren algunas cosas, todas de la misma calidad moral y que afectan de la misma manera a la dignidad, pero curiosamente están peor vistas...

(5) Usuario 17: lo peor es que acabas pensando que al menos tú tienes trabajo con un sueldo mejor que el de la mayoria... (pero al final, esto repercutirá en la calidad inevitablemente) [U.17 (h). Pub. 5]

\subsubsection{BROMAS AFILIADORAS}

Una de las formas de colaboración temática más frecuentes que expresan cortesía valorizadora son las bromas afiliadoras. Estas se repiten constantemente, y parece que existe un consenso sobre el carácter lúdico de esta plataforma. Además, influyen considerablemente en la imagen de afiliación del grupo, fortaleciendo la vinculación afectiva entre los participantes, como se ha corroborado en los ejemplos precedentes.

En este sentido, conviene recordar aquí que la confianza es considerada uno de los contenidos fundamentales de la imagen de afiliación en la cultura española. Para Hernández Flores, quien toma esta premisa de Bravo, "la confianza alude a un tipo de contrato social entre personas que supuestamente se conocen bien, lo que permite hablar o actuar abiertamente y sin miedo a ofender" (2003: 123), y en su trabajo concluye que la confianza se siente de forma general como algo positivo, siempre que se dé entre circunstancias y personas apropiadas. Por el contrario, esta autora apunta que la 
falta de confianza no conlleva una evaluación positiva dentro de un contexto de tipo familiar o entre amigos.

El siguiente ejemplo es una clara muestra de esta tendencia. Ante la publicación del usuario 14 explicando su estado de salud, además de intervenciones de ánimo (no se recogen en este fragmento), se genera una serie de intervenciones humorísticas que resta importancia al contenido del enunciado e incide en la relación de confianza y cercanía de los amigos que intervienen como respuesta a la publicación del usuario.

[6]

(1) Usuario 14: 3er urbason. Tranquilos que estoy bien!! Ingresado en observación. Adios al finde...

(...)

(10) Amigo 8 (h): Lo que hay que hacer para llamar la atención.........

(11) Amigo 9 (h): Me pido tu colección de la hora chanante y si estás moribundo es posible que acepte tb algún cd de los piratas, por hacerte feliz!!

(12) Amigo 7 (h): asi da gusto los buenos amigo dispuestos a ayudar. XD XD XD XD. yo me quedo con los comics

(13) Amigo 9 (h): si es que ya lo dice en los paquetes: "el tabasco mata"....por favor no me denuncies por el chiste....

(...)

(16) Amigo 11 (h): qué putada, nene, en qué hospital estás?

(17) Amigo 11 (h): Están buenas las enfermeras?

(18) Usuario 14: Na de na (alude a amigo 11), no merece la pena.... Hospital san carlos $\bullet$

(...)[U.14 (h). Pub. 2]

La bromas afiliadoras operan, además, ejerciendo una distinción de significado dentro del polisémico concepto de "amigo" y muestran a los participantes como personas con un lazo de mayor intimidad con el usuario que simples conocidos. En consecuencia, actúanestableciendo una relación de jerarquía de mayor a menor grado de confianza y familiaridad.

Desde otro enfoque, las publicaciones de tono humoristico de los usuarios en su muro favorecen positivamente su propia imagen, ya que los presentan como personas ingeniosas y ocurrentes; las colaboraciones temáticas hacia este tipo de enunciados efectuadas por los amigos refuerzan, por una parte, la imagen del usuario, y por la otra, la imagen de los propios participantes que ratifican o completan la publicación inicial. Un ejemplo de esta tendencia se manifiesta en la siguiente publicación:

[7]

(1) Usuario 18: Las autoridades sanitarias advierten de que ver Telecinco puede hacerte llorar sangre. 
(2) Amigo 1 (h): uooooo... eso lo tengo que probar... los efectos son reversibles? o te conviertes en un mix de belen esteban y jesucristo para la eternidad?[U.18 (h). Pub. 5]

\subsection{OTROS INDICADORES DE CORTESÍA VALORIZADORA. LA UTILIZACIÓN DE "ME GUSTA"}

Aunque no se trata de un recurso verbal, uno de los patrones de interacción más utilizados en esta red social, y que está suplantando cada vez más otras interacciones verbales, es la utilización del botón "me gusta". La interpretación de este "me gusta" no siempre responde a su significado literal, lo que implica que a menudo resulte bastante escurridizo.

Su uso está plenamente convencionalizado por parte de los usuarios de esta red social y se emplea incluso en casos donde puede resultar bastante ambiguo. Sin embargo, este recurso obedece siempre a estrategias de cortesía y posiblemente sea esta asociación lo que contribuye a que su interpretación en determinados contextos resulte positiva por parte delos usuarios (como sucede cuando se interpreta como una manifestación de empatía, apoyo o ánimo).

A continuación, se ofrecen algunos ejemplos cuyo probable significado atiende a distintas estrategias de cortesia valorizadora:

\begin{tabular}{|c|c|}
\hline $\begin{array}{l}\text { A) MANIFESTACIÓN DE } \\
\text { ALEGRÍA POR LOS PLA- } \\
\text { NES DEL USUARIO }\end{array}$ & $\begin{array}{l}\text { LA SEMANA QUE VIENE ME VOY AL CAMPO A PERDERME DEL TÓ. } \\
9 \text { DÍAS COMO UN HERMITAÑO. MUCHO PASEO, SENDERISMO, } \\
\text { LECTURA. Y LO MEJOR DE TODO SIN ORDENADOR, FACEBOOK, } \\
\text { MÓVIL, ELECTRICIDAD, ETC... A Usuario } 20 \text { y } 12 \text { personas más les gusta } \\
\text { esto.[U. 20. Pub. } 3 \text { (1) (h)] }\end{array}$ \\
\hline B) FELICITACIONES & $\begin{array}{l}\text { On Holidays. Las más esperadas y las más merecidas. :) A } 3 \text { personas les } \\
\text { gusta esto.[U.16. Pub. } 1 \text { (1) (h)] }\end{array}$ \\
\hline C) AGRADECIMIENTOS & $\begin{array}{l}\text { Felicidades, que tengas un día maravillosoooooooooo.Me gusta } 1 \text { [U. } 11 \text {. } \\
\text { Pub. } 2(10)(\mathrm{m})] \\
\text { Felicidadesss joven!!!! Cndo hechemos el próximo partidito de padel...te doy } \\
\text { un tirón de orejas. Feliz dia, bsos a la family.Me gusta } 1 \text { [U. 13. Pub. } 3 \text { (6) } \\
\text { (h)] }\end{array}$ \\
\hline $\begin{array}{l}\text { D) MANIFESTACIONES DE } \\
\text { EMPATÍA (APOYO Y } \\
\text { ÁNIMO) }\end{array}$ & $\begin{array}{l}\text { Cenar estudiando el Plan Nacional I+D+i } 2008-2011 \ldots \text { que vida mas triste. } \\
\text { A } 2 \text { personas les gusta esto.[U. 14. Pub. } 6(1)(\mathrm{h})] \\
\text { Bueno, por fin el diagnóstico es definitivo. ROTURA DEL } 2^{\circ} \text { METATARSO } \\
\text { DEL PIE IZQUIERDO. } 1 \text { MES CON ESCAYOLA. LOS } 3 \text { PRIMEROS DÍAS } \\
\text { SIN APOYAR EL PIE. POR LO MENOS SOLO SON } 3 \text { DÍAS SIN APOYAR. } \\
\text { ASÍN QUE A RECUPERARSE TOCA. A } 3 \text { personas les gusta esto.[U. } 20 . \\
\text { Pub. } 6 \text { (1) (h)] }\end{array}$ \\
\hline
\end{tabular}




\section{E) MANIFESTACIÓN DE} ACUERDO ANTE EL ENUNCIADO DE LA PUBLICACIÓN ya no me quedan adjetivos descalificativos posibles para el señor WERT. A 5 personas les gusta esto.[U. 4. Pub. 4 (1) (m)]

Si tuviese algo inteligente que decir estaría escribiendo un libro de éxito en vez de una actualización de mi estado de Facebook. A 12 personas les gusta esto.[U. 18. Pub. 1(1) (h)]

Hace cuatro años ZP llamaba a la crisis “desaceleración”..., ayer Rajoy llamaba al rescate bancario "línea de crédito"... para que luego digan los políticos que llaman a las cosas por su nombre. A 18 personas les gusta esto.[U. 10. Pub. 3 (1) (m)]

TABLA 2. EJEMPLOS DE UTILIZACIÓN PRAGMÁTICA DEL BOTÓN “ME GUSTA"

La utilización de esta herramienta resulta tan recurrente que a veces los usuarios de Facebook hacen referencias de tipo metalingüístico al uso de la misma, como reflejan los siguientes ejemplos:

[8]

Siento deciros que No Me Gusta,

ME ENCANTAAAAAAAAAAAAAAAAAAAAAAAAAAAAAAAAAAAAAA [U.10. Pub. 1 (16) (m)]

[9]

(...)

(4) Amigo 4 (m): NO me gusta:/

(5) Usuario 17: (falta el botón de "Me gusta que no te guste, jeje)[U.17 (h). Pub.3]

El hecho tan frecuente de acudir a esta opción resulta bastante útil desde el punto de vista pragmático, como señala Maíz-Arévalo refiriéndose a este uso como respuesta a los cumplidos: "The complimentee does not waste any time at all to type an answer while politely acknowledging both the reception and the appreciation for the compliment" (2013: 64). Los usuarios suelen coincidir en que su utilización sirve para indicar que el mensaje ha sido leído y al mismo tiempo reproducir un comportamiento cortés; por otra parte, permite actuar bajo el principio de economía del lenguaje garantizando a su vez la cordialidad entre los participantes.

\section{CONCLUSIONES Y FUTURAS LÍNEAS DE INVESTIGACIÓN}

1. En el caso de la red social Facebook, la función valorizadora de la cortesía parece constituirse como el eje principal de las relaciones interpersonales. A diferencia de otras redes sociales (como puede ser Twitter en España), en el corpus analizado los usuarios exhiben un uso constante de estrategias de cortesía que suponen un refuerzo para la imagen de los destinatarios, cuestión que, al mismo tiempo, repercute positivamente en su propia imagen. 
2. Una de las razones que parece propiciar la aparición de estos usos corteses en oposición a otros canales de comunicación de internet es la ausencia de anonimato y de distanciamiento entre los amigos de esta red social, unido al hecho de que las publicaciones permanecen en el muro por tiempo indefinido.

3. El concepto de "amigo" que utiliza esta red social para referirse a todo tipo de personas asociadas al perfil de un usuario parece influir en el comportamiento comunicativo de los participantes. Así, condiciona la frecuencia de manifestaciones de cortesia positiva "ritualizadas" (aquellas definidas como estrategias de cortesía directa, como cumplidos, felicitaciones, muestras de ánimo, apoyo, etc.), pues se trata de actos de habla inherentemente corteses y que no suponen ningún riesgo de amenaza para la imagen del oyente.

4. Las colaboraciones temáticas se constituyen como muestras de cortesía indirecta destinadas a cooperar con las publicaciones de los usuarios del muro; en este corpus, las más frecuentes son las manifestaciones de interés y de acuerdo y las bromas afiliadoras.

5. Desde el punto de vista pragmático, un hecho que da muestra de que la función principal de esta red social es el mantenimiento cordial de las relaciones interpersonales es el uso del botón "me gusta". La frecuencia con que los usuarios utilizan esta herramienta, sus funciones y su utilidad pragmática constituye otra cuestión que puede ser abordada en investigaciones posteriores.

6. Una valoración de tipo cuantitativo que recoja indicaciones de frecuencia referidas a las variables de sexo o condicionantes del contexto comunicativo se considera de gran interés para futuros análisis.

\section{REFERENCIAS}

ALBELDA, M. (2003): "Los actos de refuerzo de la imagen en la cortesía peninsular", Bravo, D. (Ed.): Actas del Primer Coloquio EDICE. La perspectiva no etnocentrista de la cortesia: identidad sociocultural de las comunidades hispanohablantes, Universidad de Estocolmo, Libro-e, pp. 298-305.

ALBELDA, M. (2004): "Cortesía en diferentes situaciones comunicativas: la conversación coloquial y la entrevista sociológica informal", Bravo, D. y A. Briz (Eds.): Pragmática sociocultural. Estudios sobre el discurso de cortesía en español, Barcelona: Ariel, pp. 109-134.

ALBELDA, M. (2005): La intensificación en el español coloquial. Tesis Doctoral, Valencia: Universidad de Valencia.

BARROS GARCÍA, M. J. (2011): La cortesía valorizadora en la conversación coloquial española: estudio 
pragmalingüistico. Tesis Doctoral, Granada: Universidad de Granada.

BARROS GARCÍA, M. J. (2012): "Cumplidos y ofrecimientos: actividades de cortesía valorizadora en la conversación coloquial española", Escamilla Morales, J. y G. Henry Vega (Eds.): Miradas multidisciplinares a fenómenos de cortesía en el mundo hispánico, Universidad del Atlántico y Programa Edice. Libro-e, pp. 108143.

BAYRAKTAROGLU, A. (1991): "Politeness and interactional imbalance", International Journal of the Sociology of Language, 92, pp. 534.

BERNAL, M. (2007): Categorización sociopragmática de la cortesía y la descortesía. Un estudio de la conversación coloquial española, Estocolmo: Universidad de Estocolmo.

BERNAL, M. (2005): "Hacia una categorización sociopragmática de la cortesía, la descortesía y la anticortesía. El caso de conversaciones españolas de registro coloquial", Bravo, D. (Ed.): Estudios de la (des)cortesía en español. Categorias conceptuales y aplicaciones a corpora orales y escritos, Estocolmo-Buenos Aires: Dunken, pp. 365-398.

BRAVO, D. Y A. BRIZ (Eds.) (2004): Pragmática sociocultural. Estudios sobre el discurso de cortesía en español, Barcelona: Ariel.

BROWN, P. Y S. LEVINSON (1987): Politeness: Some Universals in Language Usage, Cambridge: Cambridge University Press.

BROWN, P. Y S. LEVINSON (1978): "Universals in Language Use: Politeness Phenomena", Goody, E. (Ed.): Questions and Politeness. Strategies in Social Interaction, Cambridge: Cambridge University Press, pp. 56-289.
CAMBRA GONZÁLEZ, T. (2009): "Facebook y los trastornos de la media(ción) tecnológica en la socialización", IV Congreso de la Cibersociedad, texto completo disponible en: [http://www.cibersociedad.net/congres2009/es/coms/facebook-ylos-trastornos-de-la-mediaciontecnologica-en-la-socializacion/607/] (fecha de consulta 15/ 11/ 2013).

CARRASCO SANTANA, A. (1999): "Revisión y evaluación del modelo de cortesía de Brown y Levinson", Pragmalingüistica, 7, pp. 1-44.

GOFFMAN, E. (1959): The presentation of the Self in Everyday Life, Nueva York: Doubleday.

GOFFMAN, E. (1967): Interaction ritual: Essays on Face-to-Face Behavior, Nueva York: DoubledayAnchor.

HAVERKATE, H. (1994): La cortesía verbal. Estudio pragmalingüístico, Madrid: Gredos.

HERNÁNDEZ FLORES, N. (2003): "Cortesía y contextos socioculturales en la conversación de familiares y amigos", Bravo, D. (Ed.): Actas del primer coloquio EDICE. La perspectiva no etnocentrista de la cortesia: identidad sociocultural de las comunidades hispanohablantes, Universidad de Estocolmo. Libro-e, pp. 121-127.

HERNÁNDEZ FLORES, N. (2004): "La cortesía como la búsqueda del equilibrio de la imagen social", Bravo, D. y A. Briz (Eds.): Pragmática sociocultural. Estudios sobre el discurso de cortesía en español, Barcelona: Ariel, pp. 95108.

KERBRAT-ORECCHIONI, C. (1992): Les interactions verbales, 2 vols, Paris: Armand Colin.

KERBRAT-ORECCHIONI, C. (1997): "A multilevel approach in the study of talk-in-interaction", Pragmatics, 7, pp. 1-18. 
KERBRAT-ORECCHIONI, C. (2004): “¿Es universal la cortesía?”, Bravo, D. y A. Briz (Eds.): Pragmática sociocultural. Estudios sobre el discurso de cortesía en español, Barcelona: Ariel, pp. 3953.

LAKOFF, R. (1973): "The logic of politeness or, minding your $\mathrm{p}$ 's and $\mathrm{q}$ 's", Papers from the ninth regional meeting, Chicago: Linguistic Society, pp.292-305.

LANDONE (2012): "Discourse markers and politeness in a digital forum in Spanish", Journal of Pragmatics, 44, pp. 1799-1820.

LAVANDERA, B. (1988): "The Social Pragmatics of Politeness Forms", Ammon, U. y H. Dittmar (Eds.): Sociolinguistics. An International Handbook of the Science of Language and Society, BerlinNueva York, pp. 1196-1205.

LEECH, G. (1983): Principles of Pragmatics, Londres: Longman.
LEECH, G. (2007): "Politeness: Is there an East-West divide?", Journal of Politeness Research, 3, pp. 167-206.

MAÍZ-ARÉVALO, C. (2013): "Just click 'Like": $\quad$ Computer-mediated responses to Spanish compliments", Journal of Pragmatics 51, pp. 47-67.

PANO, A. (2008): Dialogar en la red. La lengua española en chats, $e$ mails, foros y blogs, Berlín: Peter Lang.

SIFIANOU, M. (1995): "Do we need to be silent to be extremely polite? Silence and FTAs", International Journal of Applied Linguistics, 5 (1), pp. 95-110.

TERKOURAFI, M. (2003): "Beyond the micro-nivel in politeness research", Journal of Politeness Research, 1 (2), pp. 237-262.

YUS, F. (2010): Ciberpragmática 2.0. Nuevos usos del lenguaje en Internet, Barcelona: Ariel. 